\section{Review: Psychotherapy for adolescents with depression: initial but no sustainable benefits}

\section{QUESTION}

Question: Is psychotherapy an effective way to treat children and adolescents with depression?

Outcomes: Risk of response, defined as score below the threshold for diagnosis of depression on whichever scale the study used-"operationalized criteria" or "a validated depression severity measure". Secondary outcomes were cost and safety of treatment.

\section{METHODS}

Design: Systematic review with meta-analysis.

Data sources: Cochrane CENTRAL, MEDLINE, CINAHL, PsycINFO, EMBASE, PSYNDEX, LILACS, conference proceedings and hand searches of journals; performed by searching the Cochrane Collaboration Depression, Anxiety and Neurosis Registers on 17 December 2004. Additional supplementary search of CENTRAL, MEDLINE, PsycINFO and EMBASE was carried out, and from the selected papers, reference lists were examined and lead authors contacted for further data on other trials.

Study selection and analysis: Individual or cluster randomised controlled trials of any psychotherapy (PT) versus no treatment, attention-placebo, waiting-list control, or treatment as usual, in adolescents (aged 6-18 years) with depression or dysthymia. Attention-placebo was defined as interventions that involved time spent with the patient but which did not focus on depressive symptoms (for example, psycho-drama or art therapy). Exclusions: psychotherapy that did not focus specifically on the individual-for example, family therapy, counselling. Standardised data extraction forms were used and methodological quality assessed using the Quality Rating Scale. Results from individual studies were combined and number needed to treat and relative risk of response (primary outcome) were calculated using a random effects model. Intracluster correlation coefficients were used, where available, to estimate effect size in cluster randomised trials. Subgroup analysis was planned a priori to assess the effect of psychotherapy type, control condition used, severity of depression, and age. Dropouts were included in intentionto-treat analysis (ITT).

\section{MAIN RESULTS}

Twenty seven studies involving 35 randomised comparisons (3 cluster) and 1744 patients, met the inclusion criteria. Psychotherapy (25 trials investigating cognitive behavioural therapy (CBT), 2 cognitive therapy (CT), 3 behavioural therapy (BT), 2 interpersonal therapy (IPT), 1 problem solving therapy (PST), 1 supportive therapy (ST), and 1 other) were compared to treatment as usual (TAU; used in 3 comparisons and included any non-study mental health care), attentionplacebo (8 comparisons; including health education and psychodrama), waiting list control (18 comparisons), and no treatment (6 comparisons). Psychotherapy improved posttreatment response compared to control overall: 450/907 (49.6\%) of the PT group had responded at study completion compared to $267 / 767$ (34.8\%) of the control groups (RR response: $1.39,95 \%$ CI 1.18 to $1.65 ; p=0.0001)$. Estimated NNT was 4.3. In analyses specifically looking at 1-6 month follow-up and 6-12 month follow-up, there were no differences between psychotherapy and the control conditions ( $p>0.15$ for both). For subgroup analyses see online notes.

\section{CONCLUSIONS}

Psychotherapy improves post-treatment response for children and adolescents with depression compared to control, with most evidence for CBT. However, adding PT to usual treatment for depression does not improve response above usual treatment alone. Benefit is greatest for those in the 1218 age group. Benefit over control is not sustained at longerterm follow-up.

\section{ABSTRACTED FROM}

Watanabe N, Hunot V, Omori IM, et al. Psychotherapy for depression among children and adolescents: a systematic review. Acta Psychiatr Scand 2007;116:84-95.

Correspondence to: Norio Watanabe, Department of Psychiatry and Cognitive Behavioral Medicine, Nagoya City University Graduate School of Medical Sciences, Mizuho-cho, Mizuho-ku Nagoya 467-8601, Japan; norio@med.nagoya-cu.ac.jp

Source of funding: The Cochrane Child Health Field Bursay Scheme.

- Additional notes and a reference list are published online only at http:// ebmh.bmj.com/content/vol11/issue2
$\mathrm{T}$ his rigorous review addressed the issue of the effectiveness of psychotherapy in the treatment of depression in children and adolescents. It is worth noting that the review includes a particularly large study on targeted depression prevention $^{1}$ which excluded young people with depressive disorder, thus reducing the chance of showing an effect for psychotherapy.

The results provide evidence that cognitive behaviour therapy (CBT), interpersonal therapy (IPT) and possibly behaviour therapy (BT) are effective, at least for adolescents. The response rates on metaanalysis (49.6\% psychotherapy vs $34.8 \%$ control) compare well with antidepressants. ${ }^{2}$ The psychotherapy studies have used self-rating scales of depressive symptoms. Studies of antidepressants have used clinician rated scales as primary outcomes and most have failed to show a difference on self-rating scales (using similar measures). ${ }^{2}$ Had clinician rating scales been used the results from psychotherapy might have been even better.

A difference in outcome at short-term follow-up (1-6 months) would have been expected with effective therapy, and failure to show this is of some concern, while the failure to show a difference at longer term follow-up (6-12 months) may well reflect a catch-up from spontaneous remission in the comparison group.

The authors rightly identify weaknesses in the current studies and point out the lack of information on cost-effectiveness and on the potential for harm. However, until more rigorous studies are done this review supports the continued use of CBT and IPT, and probably BT for depression in children and adolescents.

The challenge remains. Neither psychotherapy nor medication $^{2}$ result in high rates of remission for depressive disorder. The quest for more effective therapies goes on. In the meantime, this review provides evidence to support the continued use of CBT and IPT in depression in adolescents, in line with the NICE guidelines. ${ }^{3}$ The issue is less clear for children.

Sally N Merry, MB, ChB, FRANZCP, MD Werry Centre for Child and Adolescent Mental Health, University of Auckland, Auckland, New Zealand

Competing interests: None. 